\title{
Crack detection and filling, using steepest descent method
}

\author{
I. A. Ismail, E. A. Rakh, S. I. Zaki, M. A. Ashabrawy, M. K. Shaat
}

\begin{abstract}
An integrated methodology for the detection and removal of cracks on digitized images is presented in this paper. The cracks are detected by steepest descent algorithm (SDA) the output of the piping image as cracks are removed using either a gradient Function (GRF) and processed data or a semi-automatic procedure based on region growing. Finally, crack filling using the steepest descent algorithm. The methodology has been shown to perform very well on digitized images suffering from cracks, using Matlab, Surfer and Visual Fortran programming.
\end{abstract}

Key words - crack detection, filling cracks, steepest descent.

\section{INTRODUCTION}

The steepest descent algorithm is an old mathematical tool for numerically finding the minimum value of a function, based on the gradient of that function. Steepest descent uses the gradient function (or the scalar derivative if the function is single-valued) to determine the direction in which a function is increasing or decreasing most rapidly. Each successive iteration of the algorithm moves along this direction for specified step size and the recomputed the gradient to determine the new direction to travel [1].

Digital image processing analysis and computer visions have exhibited an impressive growth in the past decade in terms of both theoretical development and applications. They constitute a leading technology in a number of very important areas, for example in digital telecommunication, broadcasting medical imaging. multimedia systems, biology, material sciences, Robotics and manufacturing, intelligent sensing systems, remote sensing, graphic arts and printing[2], [3] and [4] and [5].

The rest of the paper is organized as follows. In Section 2, the required computations are presented steepest descent algorithm and contains the description of our algorithm (SDA). the implementation of the algorithm is illustrated in Section 3. Statistical analysis is illustrated in Section 4. The conclusions and further work are given in Section 5.

\section{STEEPEST DESCENT ALGORITHM ( SDA)}

The gradient is a vector operator denoted $\nabla$ : $\nabla f=\operatorname{grad}(f)$ the gradient is given by:

$$
\nabla f(x, y, z)=\frac{1}{h_{1}} \frac{\partial f}{\partial x} \hat{x}+\frac{1}{h_{2}} \frac{\partial f}{\partial y} \hat{y}+\frac{1}{h_{3}} \frac{\partial f}{\partial z} \hat{z}
$$

The direction of $\nabla f$ is the orientation in which the directional derivative has the largest value and $|\nabla f|$ is the value of that directional derivative. Furthermore, if $\nabla f \neq 0$, then the gradient is perpendicular to the level curve through $\left(x_{0}, y_{0}\right)$ if $z=f(x, y)$ and perpendicular to the level surface through $\left(x_{0}, y_{0} z_{0}\right)$ if $f(x, y, z)=0$. We can generalized the equation ( 1 ) for $x_{k}$ where $k=1,2,3, \ldots \ldots \ldots, n$

$\nabla f(\vec{x})=\left\{\frac{\partial f(\vec{x})}{\partial x_{1}}, \frac{\partial f(\vec{x})}{\partial x_{2}}, \ldots+\frac{\partial f(\vec{x})}{\partial x_{n}}\right\}$

We applying this function on file of data for the piping image of $f\left(x_{1}, x_{2}, x_{3}\right)$, recall that the gradient vector in (2) points locally in the direction of great rate of increase of $f(\vec{x})$. Hence $-\nabla f(\vec{x})$ points locally in the direction of greatest decrease $f(\vec{x})$. Start at the point $\vec{p}_{0}$ and search along the line through $\vec{p}_{0}$ in the direction $\vec{s}_{0}=-\nabla f\left(\vec{p}_{0}\right) /\left\|-\nabla f\left(\vec{p}_{0}\right)\right\|$. you will arrive at a point $\vec{p}_{1}$, where a local minimum occurs when the point $\vec{x}$ is constrained to lie on the line $\vec{x}=\vec{p}_{1}+v \vec{s}_{0}$. Since partial derivatives are accessible, the minimization process can be executed using either the quadratic or cubic approximation method [ 3 ], [6] and [ 7 ].

Next we compute $-\nabla f\left(\vec{p}_{1}\right)$ and move in the search direction $\vec{s}_{1}=-\nabla f\left(\vec{p}_{1}\right) /\left\|-\nabla f\left(\vec{p}_{1}\right)\right\|$. you will come to $\vec{p}_{2}$, where a local minimum occurs when $\vec{x}_{\text {is }}$ constrained to lie on the line $\vec{x}=\vec{p}_{1}+v \vec{s}_{1}$. Iterative will produce a sequence, $\left\{\vec{p}_{k}\right\}_{k=0}$, of points with the property $f\left(\vec{x}_{0}\right) \succ f\left(\vec{x}_{1}\right) \succ \ldots \succ f\left(\vec{x}_{k}\right) \succ \ldots$ $\lim \overrightarrow{\mathrm{p}}_{\mathrm{k}}=\overrightarrow{\mathrm{p}}$ then $\mathrm{f}(\overrightarrow{\mathrm{p}})$ will be a local minimum $f(\vec{x})$. $k \rightarrow \infty$

Outline of the steepest descent algorithm (SDA)

Suppose that $\vec{p}_{k}$ has been obtained.

Step 1: Evaluate the gradient vector $\nabla f\left(\vec{p}_{k}\right)$.

Step 2: Compute the search direction

$$
\vec{s}_{k}=-\nabla f\left(\vec{p}_{k}\right) /\left\|-\nabla f\left(\vec{p}_{k}\right)\right\| .
$$

Step 3: Perform a single parameter minimization of $\Phi(v)=f\left(\vec{p}_{k}+v \vec{s}_{k}\right)$ on the interval [ 0,b ], where $\mathrm{b}$ is large. This will produce a value $v=h_{\text {min }}$ where a local minimum for $\Phi(v)$. The relation $\Phi\left(h_{\text {min }}\right)=f\left(\vec{p}_{k}+h_{\text {min }} \vec{s}_{k}\right)$ shows that this is a minimum for 
$f\left(\vec{p}_{k}\right)$ along The search line $\vec{x}=\vec{p}_{k}+v \vec{s}_{k}$.

Step 4: Construct the next point $\vec{p}_{k+1}=\vec{p}_{k}+h_{\min } \vec{s}_{k}$.

Step 5: Perform the termination test for

minimization, as: Are the function value

$f\left(\vec{p}_{k}\right)$ and $f\left(\vec{p}_{k+1}\right)$ Sufficiently close and

the distance $\left\|\vec{p}_{k+1}-\vec{p}_{k}\right\|$ small enough ?

Step 6: Repeat the process.

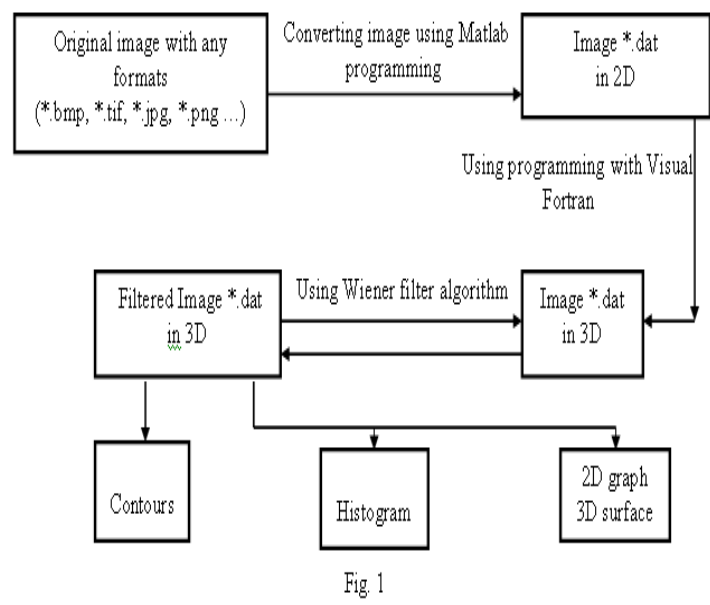

III. THE IMPLEMENTATION OF THE ALGORITHM

In this part of the paper we take two image $a$ and $b$ have cracks and these cracks increasing in image $b$, we applying the steepest descent algorithm (SDA) for detecting the cracks and filling it or removal of cracks in digitized image. We get all figures, Fig. 2. Original image for two cases shows the increasing the cracks fro a , b. Fig. 3. Analysis of Original image a .

Fig. 4. Filling the cracks in image a for two iteration i, ii. Fig. 6. Filling the cracks in image $b$ for three iteration i, ii, iii. Fig. 7. Filling the cracks in image a for vector map using (SDA). We show also 3D surface for the crack image a , b. the relation between distances pixels and gray values ( intensity of color ) in a, b images. Contours of image a Vector map using (SDA) of image $a, b$.

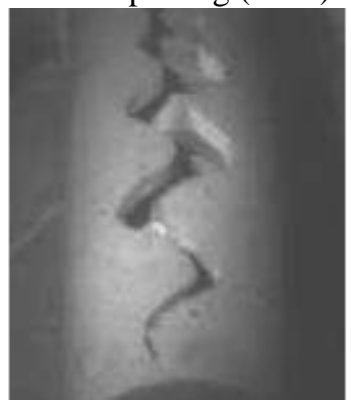

a

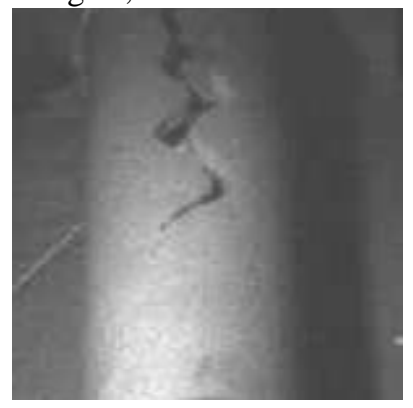

c
Fig. 2. Original image for two cases shows the increasing the cracks fro a , b

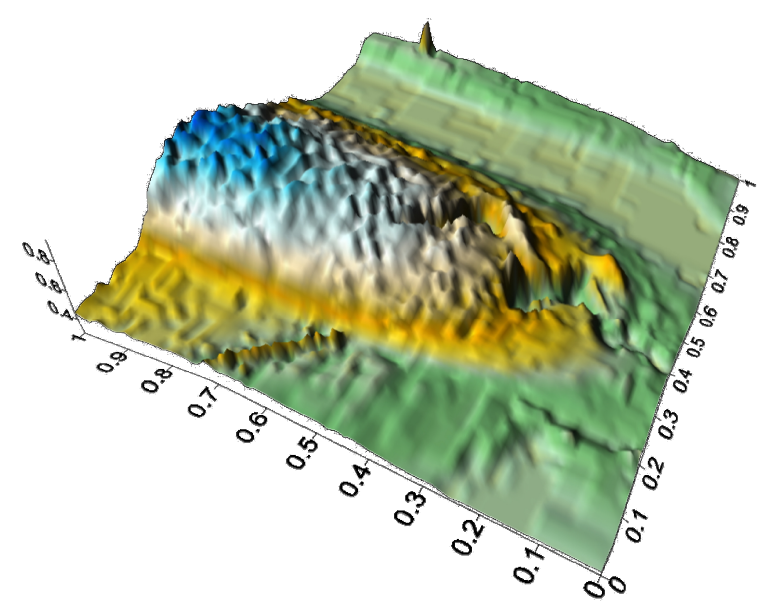

3D surface for the crack image a

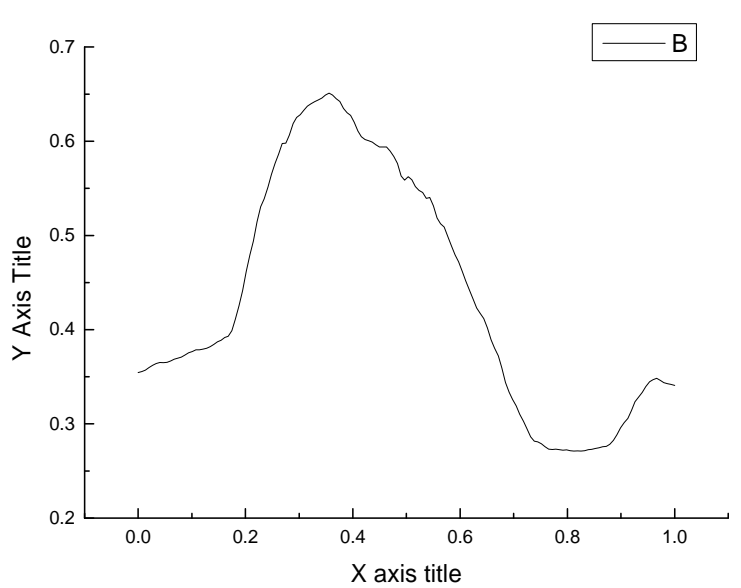

The relation between distances pixels and gray values (intensity of color)

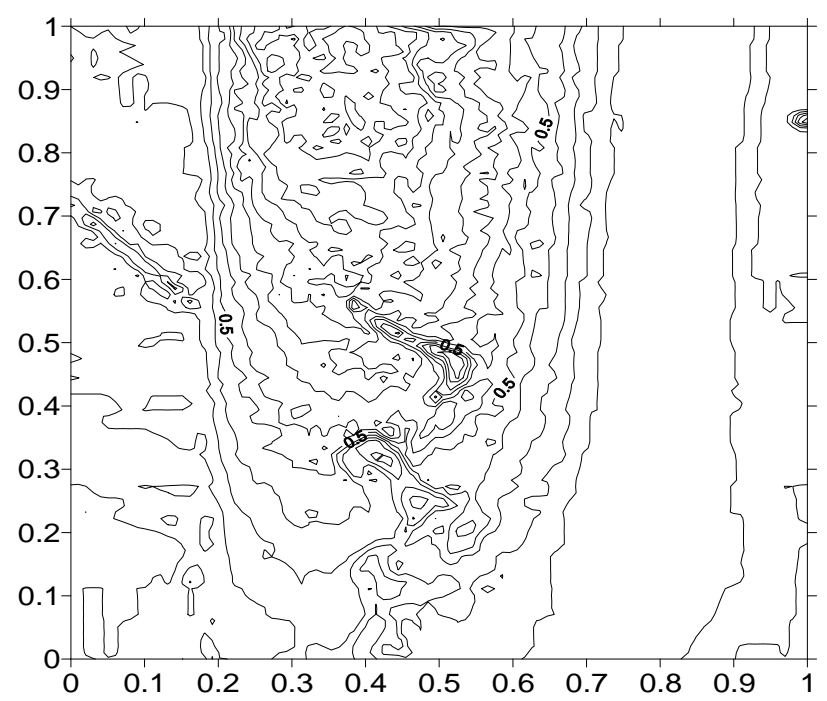

ontours of image a 


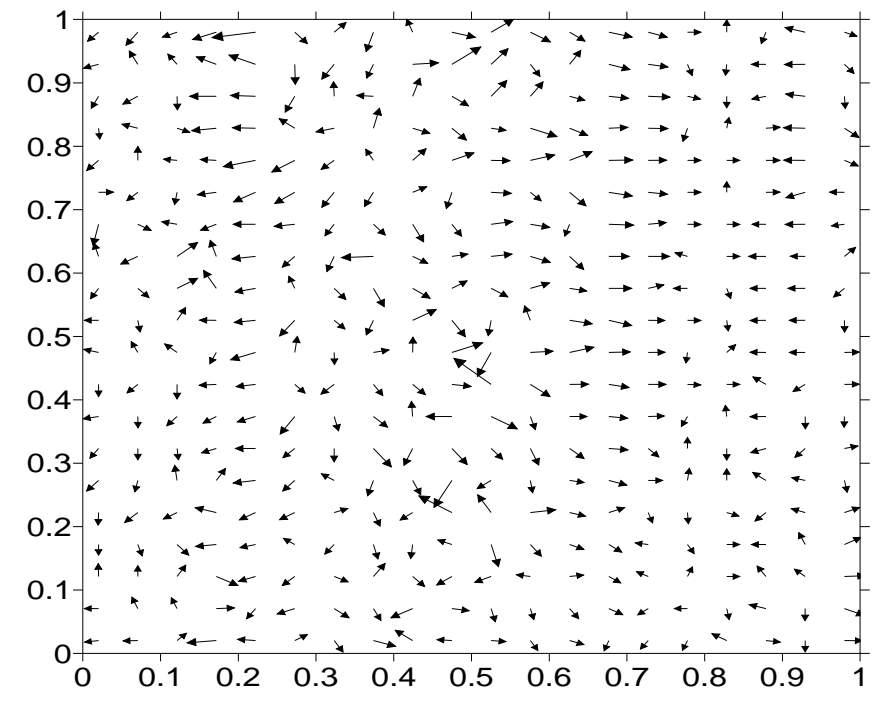

Fig. 3. analysis of Original image a Vector map using (SDA) of image a

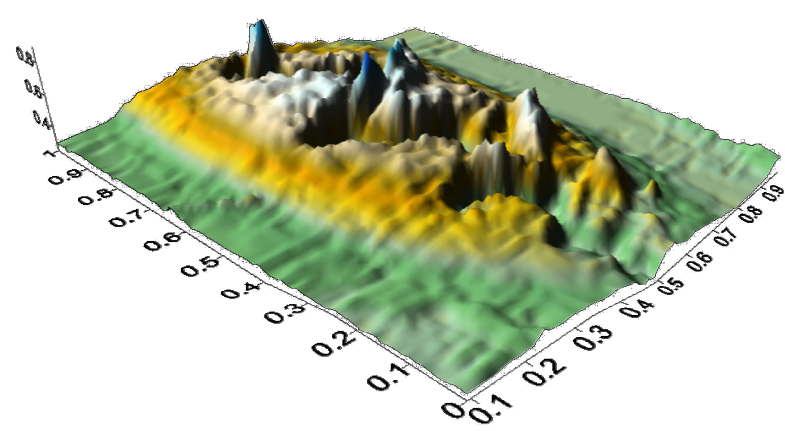

3D surface for the crack image

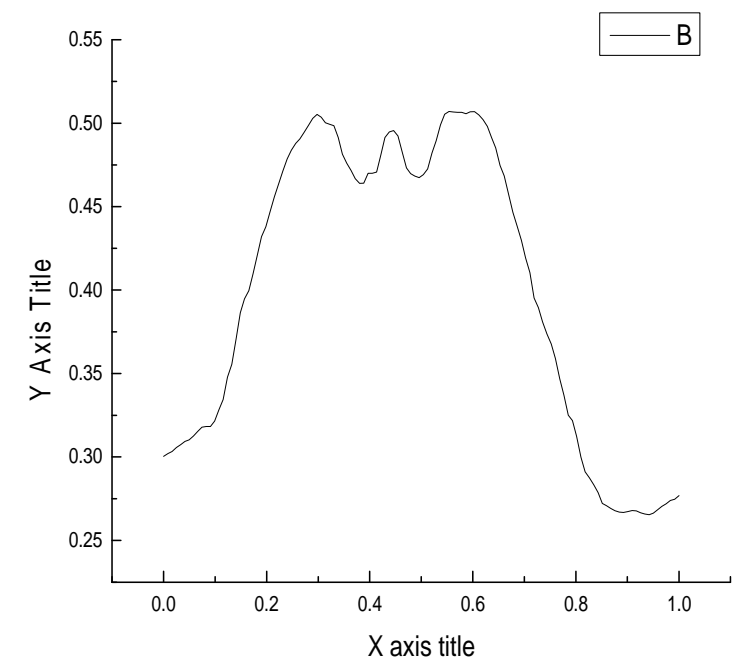

The relation between distances pixels and gray values (intensity of color)
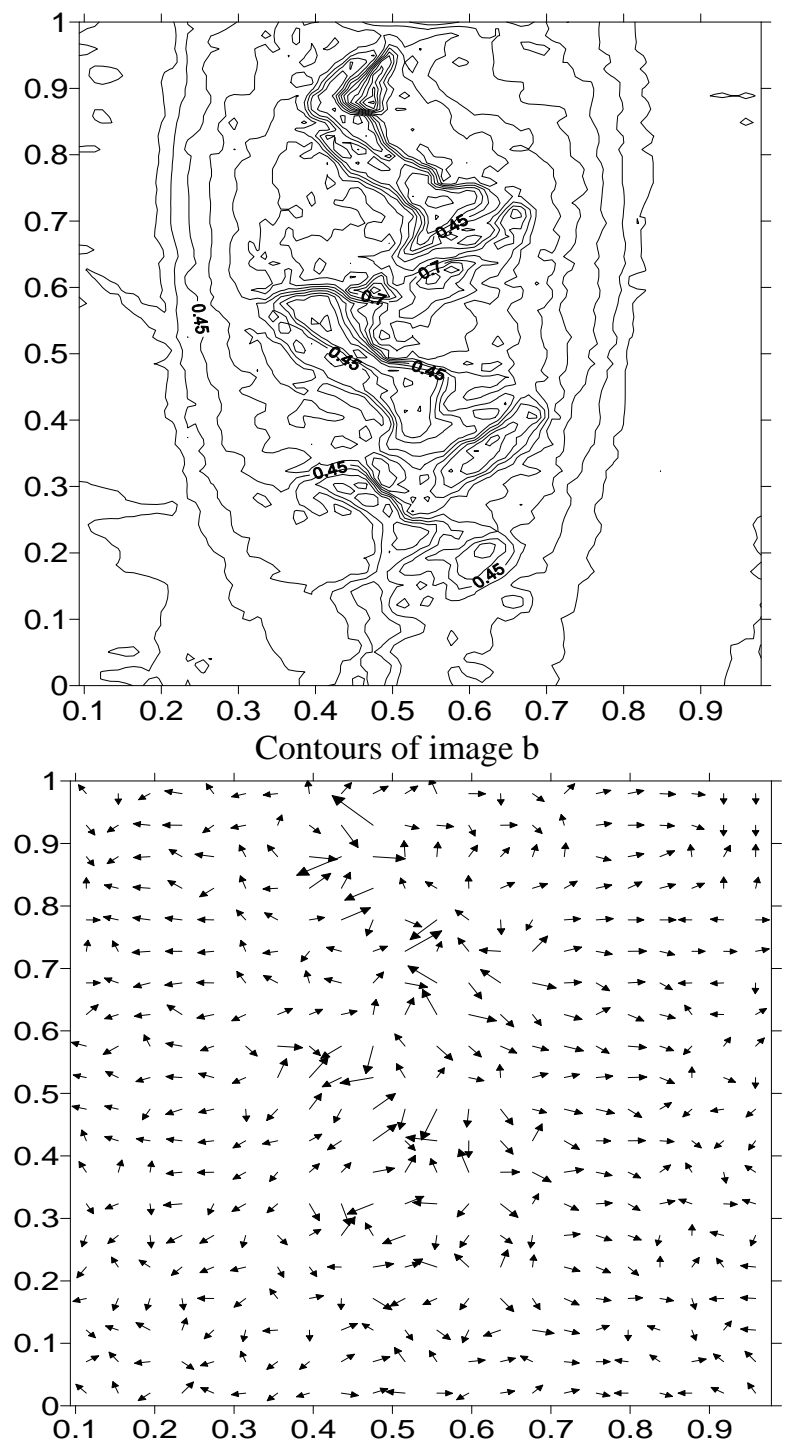

Vector map using (SDA) of image $b$ Fig. 5. analysis of Original image $b$

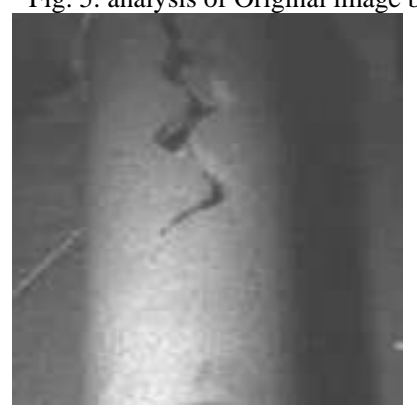

Original image a
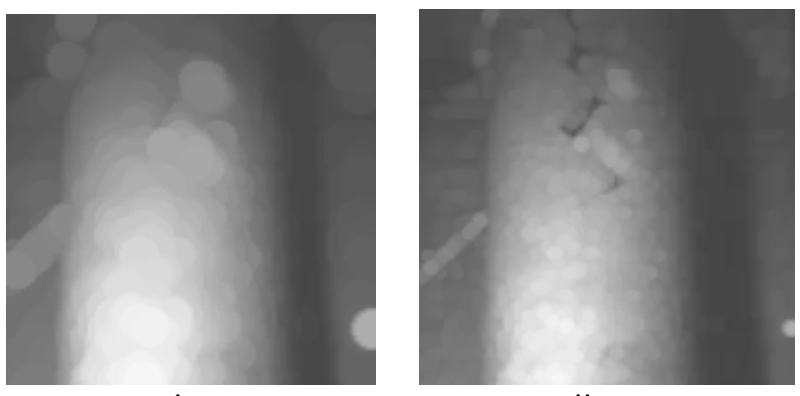

ii

Fig. 4. Filling the cracks in image a for two iteration i, ii 


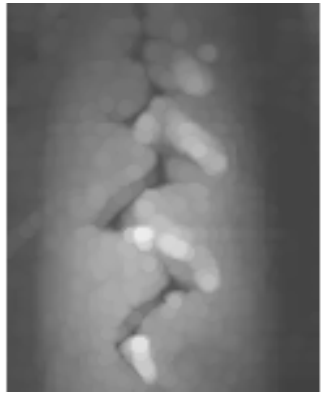

Original image $b$

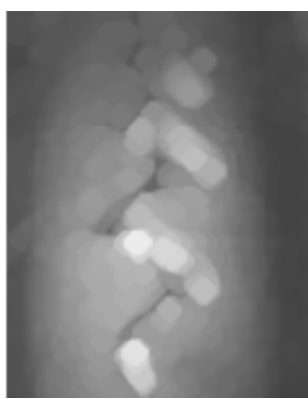

ii

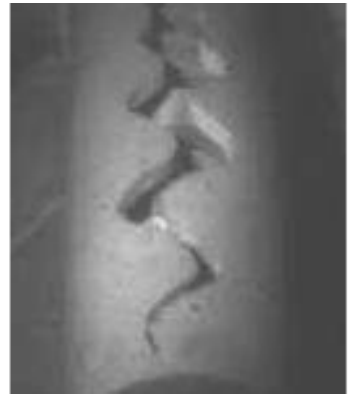

i

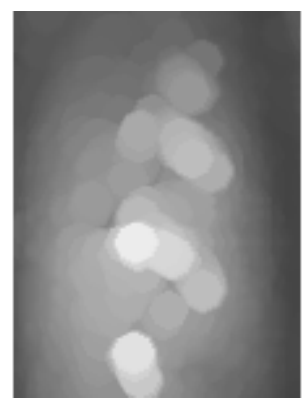

iii
Fig. 6. Filling the cracks in image $b$ for three iteration i, ii, iii

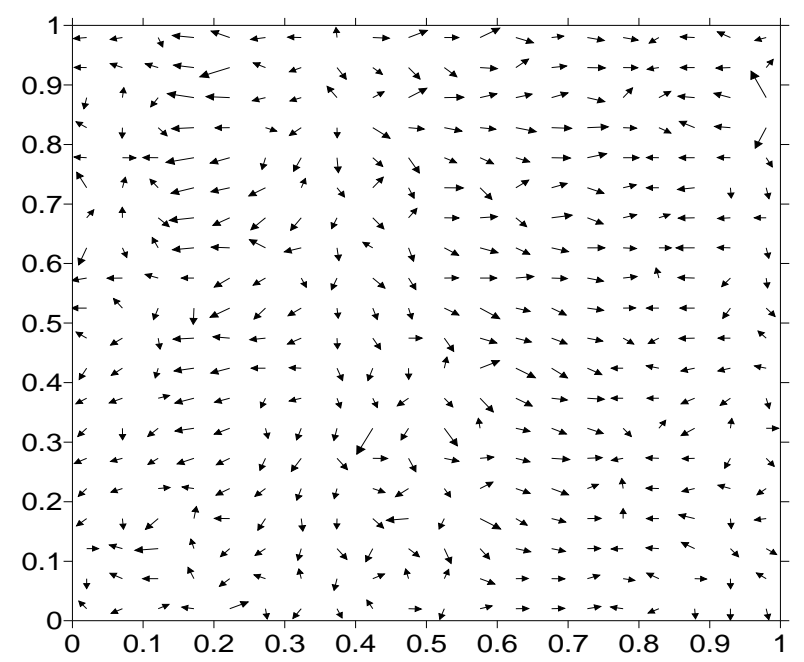

Vector map using (SDA) of image a after filling

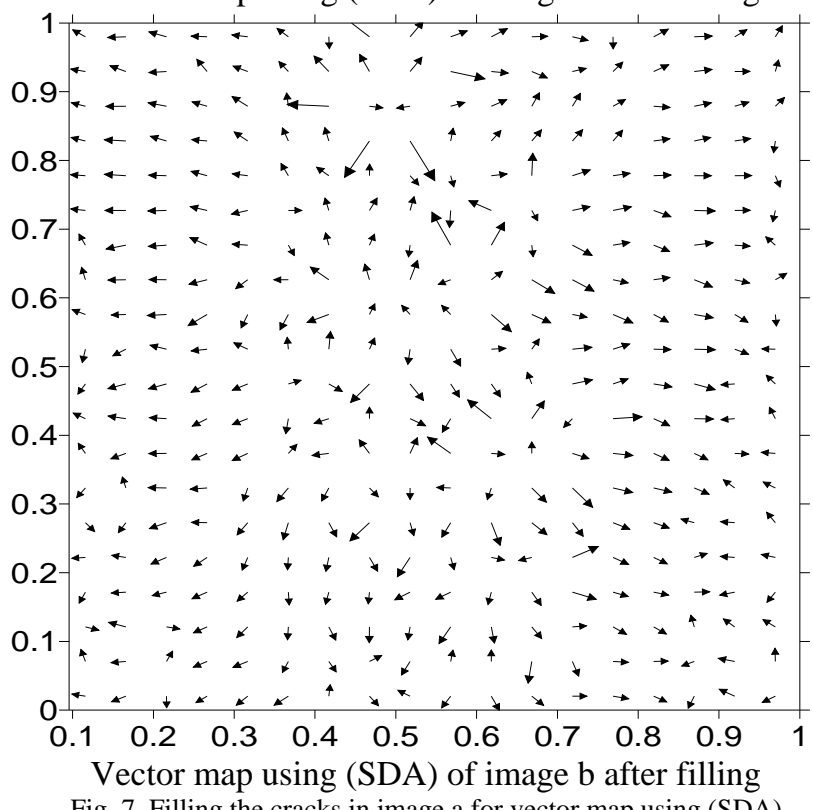

\section{CONCLUSION}

In this paper we have presented an integrated strategy for crack detection and filling in digitized piping images are detected by using steepest descent algorithm (SDA), Crack interpolation is performed by appropriately modified also by using steepest descent algorithm (SDA). We have a statistical result as mean, median, standard deviation, variance and sum of squares ( SS ) and mean square ( MS ) after detecting and filling the cracks are shown in tables above. Then the steepest descent algorithm is a useful tool for digital image processing because it can be applied iteratively.

\section{REFERENCES}

[1] Akai, Terrence J., (1994), Applied Numerical Methods for Engineers, John Wiley and Sons, Inc., New York, 410 pp.

[2] A. Rosenfeld, A. Kak, Digital Picture Processing, Academic Press, New York, 1982.

[3] F. Zana, J.C. Klein, "Segmentation of Vessel-Like Patterns Using Mathematical Morphology and Curvature Evaluation,” IEEE Transactions on Image Processing 10, pp. 1010-1019, July 2001.

[4] I. Giakoumis, I. Pitas, "Digital Restoration of Painting Cracks," in ISCAS '98, Proceedings of the IEEE International Symposium on Circuits and Signals, pp. 269-272, 31 May-3 June 1998.

[5] A.K. Jain, R.P.W. Duin, J. Mao, "Statistical Pattern Recognition: A Review," IEEE Transactions on Pattern Analysis and Machine Intelligence 22, pp. 4-37, January 2000.

[6] Ripley, B. D., (1981), Spatial Statistics, John Wiley and Sons, New York, $252 \mathrm{pp}$.

[7] Tuma, Jan J., (1979), Engineering Mathematics Handbook, 2nd edition, McGraw-HillBook Company, New York, 394 pp.

I. A. Ismail, Dean of Computer science of masir Faculty of University.

E. A. Rakh, Faculty of Science, Suez Canal University, Egypt,

S. I. Zaki, Head of mathematical department, Faculty of Science, Suez Canal University, Egypt

M. A. Ashabrawy, Department. Of Reactors, Atomic Energy Authority, Egypt.

M. K. Shaat, Department. Of Reactors, Atomic Energy Authority, Egypt. 